\title{
Glucosamine-containing supplement improves locomotor functions in subjects with knee pain - a pilot study of gait analysis
}

This article was published in the following Dove Press journal:

Clinical Interventions in Aging

20 June 2016

Number of times this article has been viewed

\author{
Noriyuki Kanzaki' \\ Yuta Otsuka' \\ Takayuki Izumo' \\ Hiroshi Shibata' \\ Hideyuki Nagao ${ }^{2}$ \\ Keita Ogawara ${ }^{3}$ \\ Hiroshi Yamada ${ }^{3}$ \\ Seiji Miyazaki ${ }^{3}$ \\ Yutaka Nakamura ${ }^{3}$ \\ 'Institute for Health Care Science, \\ Suntory Wellness Ltd, Seika-cho, \\ Soraku-gun, Kyoto, Japan; ${ }^{2}$ Research \\ Institute of Sports Medical Science, \\ Tokai University, Hiratsuka, Kanagawa, \\ Japan; ${ }^{3}$ School of Physical Education, \\ Tokai University, Hiratsuka, \\ Kanagawa, Japan
}

Correspondence: Noriyuki Kanzaki Institute for Health Care Science, Suntory Wellness Ltd, 8-I-I Seikadai, Seika-cho, Soraku-gun, Kyoto 619-0284, Japan $\mathrm{Tel}+8 \mathrm{I} 5031820666$

Fax +81774986262

Email Noriyuki_Kanzaki@suntory.co.jp
Background: Previously, we demonstrated that glucosamine-containing supplementation was effective for improving locomotor functions, especially walking speed. However, the biomechanical mechanism of efficacy has not been elucidated. This study aimed to address this challenge in subjects with knee pain, using a motion capture system.

Methods: An open label study was conducted in 30 Japanese subjects with knee pain. The subjects were administered a daily supplement containing 1,200 $\mathrm{mg}$ of glucosamine hydrochloride, $60 \mathrm{mg}$ of chondroitin sulfate, $45 \mathrm{mg}$ of type II collagen peptides, $90 \mathrm{mg}$ of quercetin glycosides, $10 \mathrm{mg}$ of imidazole peptides, $1 \mathrm{mg}$ of proteoglycan, and $5 \mu \mathrm{g}$ of vitamin D (GCQID). The intervention continued for 16 weeks. Efficacy for locomotor functions involving the knee joint was evaluated mainly using the Japanese Knee Osteoarthritis Measure (JKOM) and the 5-question Geriatric Locomotive Function Scale (GLFS-5). To examine the biomechanical mechanism of efficacy for locomotor functions, motions of subjects in a normal walking state were captured. Gait analysis was conducted and efficacy for gait parameters such as normal walking speed, stride length, cadence, and angle of soles was evaluated.

Results: GCQID significantly improved total scores on the JKOM and GLFS-5. In gait analysis, normal walking speed, stride length, and angle of soles at the end of the stance phase were all significantly increased, but cadence did not change significantly during the intervention period. There were significant intercorrelations of changes in normal walking speed, stride length, and angle of soles at the end of the stance phase, and between changes in stride length and total JKOM score.

Conclusion: A GCQID supplement may increase walking speed through increased stride length and angle of kicking from the ground during steps, which might be mainly associated with alleviated knee pain.

Keywords: locomotive syndrome, GLFS-5, normal walking speed, stride length, toe angle

\section{Introduction}

The number of elderly people aged 65 years and older has been increasing worldwide, and the aging of the population is particularly rapid in Japan. In 2007, the Japanese Orthopaedic Association proposed the concept of "locomotive syndrome" (LS), which refers to the conditions under which people require or may soon require nursing-care services because of problems with the locomotor system. ${ }^{1}$ The Japanese Ministry of Health, Labour and Welfare has devoted effort to increasing the percentage of individuals who know about LS in "The second term of National Health Promotion Movement in the 21 st Century (Health Japan 21 [second term])" to solve the problem of LS. ${ }^{2}$ Screening tools for risk of LS in the elderly, the 25-question Geriatric Locomotive Function 
Scale (GLFS-25) and the 5-question GLFS (GLFS-5), which is a quick 5-item version of the GLFS-25, were developed for early detection of $\mathrm{LS},{ }^{3}$ and have been used to raise awareness about preventing the onset of LS.

Osteoarthritis (OA) is the most common joint disease, which is one of the causes of LS and causes pain in elderly patients. According to the Research on Osteoarthritis Against Disability, more than 25 million Japanese people aged 40 years and older are estimated to have radiographic knee OA. ${ }^{4}$ Similarly, the Longitudinal Cohort of Motor System Organ study reported that the prevalence of knee pain was $32.7 \%$ in the population and that it increased with age. ${ }^{5}$ It has been suggested that knee OA impairs locomotor functions because of the pain, and the biomechanical mechanism of impaired locomotor functions such as walking speed and stride length in patients with knee OA has been investigated. ${ }^{6,7}$

Glucosamine-containing supplements have been used widely for improving knee pain in OA. ${ }^{8,9}$ In our previous studies, we demonstrated that glucosamine-containing supplements "GCQ” or "GCQID” (glucosamine hydrochloride, chondroitin sulfate, type II collagen peptides, quercetin glycosides, imidazole peptides, proteoglycan and vitamin D) were effective for relieving knee pain and/or locomotor functions in randomized, placebo-controlled trials. ${ }^{10,11}$ The effects of thermal and exercise therapies on locomotor functions and their mechanism in patients with knee OA have been elucidated with biomechanical methods, ${ }^{12}$ but the biomechanical mechanism of the effects of supplements such as GCQID on locomotor functions has not been reported.

In the present study, we used a motion capture system to investigate the biomechanical mechanism for the improving effects of GCQID on locomotor functions in subjects with knee pain.

\section{Methods}

\section{Study design and participants}

An open label study was conducted to elucidate the mechanism of efficacy of GCQID supplementation for locomotor functions in Japanese women and men aged 40 to 74 years. Inclusion criteria were the presence of knee pain, confirmed using the walking subscale of the Japanese Orthopaedic Association criteria (25 points or less for either the left or right knee joint), ${ }^{13}$ and a visual analog scale (VAS) score for knee pain (20 mm or above using the first [I] item of the Japanese Knee Osteoarthritis Measure [JKOM]); ${ }^{14}$ KellgrenLawrence grades $0-\mathrm{II} ;{ }^{15}$ and the presence of LS confirmed with a score of 6 or higher on the GLFS-5. ${ }^{3}$ All participants received an explanation about the study from the medical investigator, and written informed consent was obtained prior to enrollment in the study. Exclusion criteria were the same as in our previous study, ${ }^{11}$ and typical reasons for exclusion were the following: fast walking speed ( $\geq 1.6 \mathrm{~m} / \mathrm{s}$ ); presence of hyperuricemia, diabetes, cardiovascular disease, hepatic disease, renal disease or heart disease; presence of rheumatoid arthritis that may cause joint pain; surgical treatment of knee joint(s) needed or undergone; daily use of a cane; daily or occasional vigorous exercise; need for pharmacological articular treatments during the study period; a history of osseous or articular diseases other than OA within the past 3 months; routine use of health food or medicine that may affect the outcome of the study; and presence of any medical condition as judged by the medical investigator.

Thirty participants were enrolled in the study, which was performed from February to August 2014 at a clinical services center and at Tokai University in Japan. All subjects were recruited around the Kanto region in Japan, through a volunteer bank managed by TTC Co., Ltd (Tokyo, Japan). Once radiographs were obtained, Kellgren-Lawrence grades of subjects were determined by an orthopedic surgeon. The study protocol was approved by the ethics committee of Tokai University (Kanagawa, Japan) and the ethics committee of Tana Orthopedic Surgery (Yokohama, Japan), and was conducted in accordance with the principles of the amended Declaration of Helsinki and the Ethical Guidelines for Epidemiological Research (issued by the Japanese government in 2008).

\section{Supplementation}

The GCQID contained 1,200 mg of glucosamine hydrochloride, $300 \mathrm{mg}$ of shark cartilage extract (60 $\mathrm{mg}$ as chondroitin sulfate, $45 \mathrm{mg}$ as type II collagen peptides), $90 \mathrm{mg}$ of quercetin glycosides, $100 \mathrm{mg}$ of fish meat extract $(10 \mathrm{mg}$ as imidazole peptides [anserine and carnosine]), $5 \mathrm{mg}$ of salmon nasal cartilage extract ( $1 \mathrm{mg}$ as proteoglycan), and $5 \mu \mathrm{g}(200 \mathrm{IU})$ of vitamin D in six tablets. The GCQID tablets were manufactured by Suntory Wellness Ltd (Tokyo, Japan) specifically for the purpose of the present study, and all subjects were instructed to take six tablets once a day and to record in their study diary whether they took the tablets.

\section{Outcomes for efficacy assessment}

The indicators of efficacy of GCQID supplementation for functions of locomotor system including the knee joint were: JKOM category I score (VAS score for JKOM knee pain), 
total score for JKOM categories II-V (JKOM total score), GLFS-5 score, VAS score for knee pain in various daily situations, and questionnaires designed for this study. JKOM is a self-administered, disease-specific measure for knee OA and has demonstrated adequate validity and reliability for studies of Japanese people with knee OA in terms of outcomes correlated with health-related quality of life (QOL). ${ }^{14}$ The VAS score for JKOM knee pain was measured on a scale from 0 to 100 , where 0 indicated no pain and 100 indicated the worst pain ever experienced. The JKOM total score comprised 25 items that cover four different categories: II: pain and stiffness in knees; III: conditions in daily life; IV: general activities; and V: health conditions. Scores from the 25 items were summed, with results ranging from 0 points (no complaint) to 100 points (most severe condition possible). The GLFS-5 is also a self-administered measure, consisting of five items located in the key domain of construct structure of the GLFS-25, which covers a wide range of issues, from pain to QOL. ${ }^{3}$ Items are graded with 5-point scales from 0 points to 4 points, and the five item scores were summed to produce an overall score with a range of 0 (no impairment) to 20 points (severe impairment). The VAS score for knee pain in various daily situations included the pain in each knee on rest, walking or ascending/descending stairs, with scales from 0 to 100 , where 0 indicated no pain and 100 indicated the worst pain ever experienced. Questionnaires addressed frequency of trips that involved walking or ascending/ descending stairs, with 5-point scales from 0 (no occurrence) to 4 (frequent occurrence).

For the biomechanical mechanism of efficacy for locomotor functions, motion capture analysis in a normal walking state was conducted to obtain data on gait parameters. Subjects took off their shoes and changed into dedicated full-body suits for motion capture. Markers were attached to 30 locations on the body suits (top of head, tragi, superior margin of the sternum, extremitas inferior ribs, anterior superior iliac spines, acromions, elbows, wrists, backs of the hands, great trochanters, interior and exterior sides of knees, internal and external malleoli, heels and toes). Thereafter, subjects were instructed to walk $10 \mathrm{~m}$ two times at their usual speed. The time it took to walk the middle $6 \mathrm{~m}$ was measured and normal walking speed was calculated as the primary outcome. The position trajectories of markers attached on the suits of subjects during walking were recorded by a motion capture system (Raptor-E Digital Real Time System; Motion Analysis Corporation, Santa Rosa, CA, USA) at the same time. The values of step length, stride length, cadence, time in the stance phase and the swing phase, and angle of soles and toes for both feet were calculated by analyzing the data of position trajectories, and the average value of the data on both feet was evaluated. Explanation of terms for some parameters in motion capture analysis is shown in Figure 1.

Data on functions of locomotor system including the knee joint were collected at baseline and every 4 weeks to week 16. Data on motion capture analysis were collected at baseline and at 8 and 16 weeks. Exclusion criteria for efficacy assessment were as follows: taking the test supplement on fewer than $80 \%$ of the scheduled days, performing actions that affected the reliability of the efficacy assessment and noncompliance with the clinical protocol.

\section{Statistical analysis}

All data are expressed as the mean \pm standard error unless otherwise specified. Changes in measurements during the intervention were compared with baseline using the Steel test for JKOM total score, GLFS-5 score and questionnaires; Dunnett's test was used for the VAS score for JKOM knee pain, normal walking speed, and parameters measured by motion capture analysis. Correlation analysis between changes in parameters analyzed by the motion capture system and locomotor functions that involve the knee joint over the 16-week intervention period was conducted, and Pearson's correlation coefficient ( $r$ value) for parametric variables and Spearman's rank correlation coefficient ( $\rho$ value) for nonparametric variables were calculated. $P$-values less than 0.05 were considered significant. All statistical analyses
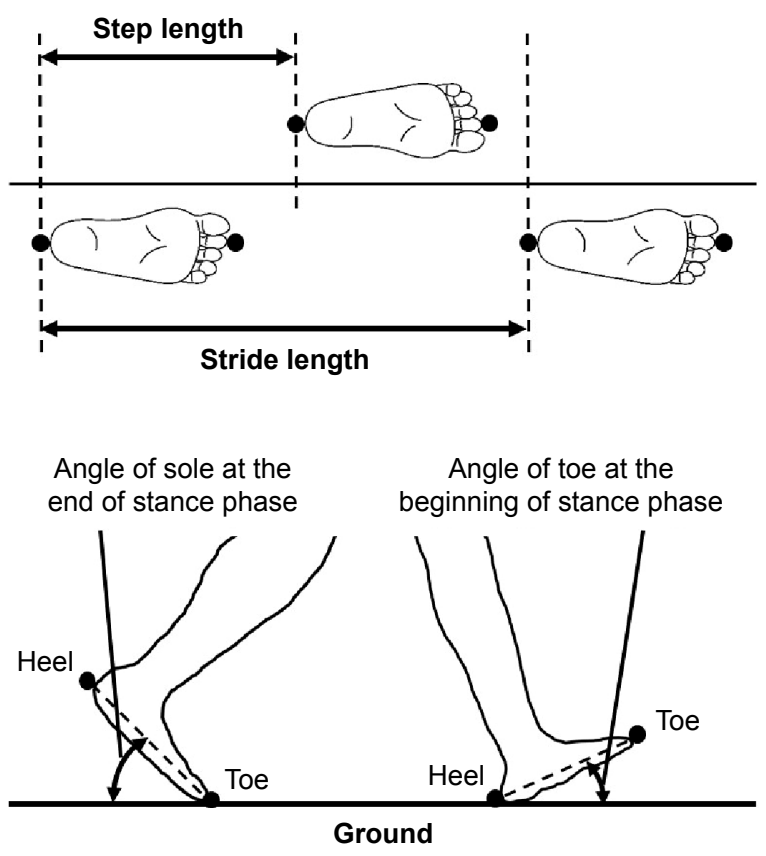

Figure I Diagram of motion capture analysis parameters. 
were carried out using IBM SPSS Statistics for Windows, Version 21.0 (IBM Corporation, Armonk, NY, USA) and Ekuseru-Toukei 2010 for Windows (Social Survey Research Information Co. Ltd, Tokyo, Japan).

\section{Results}

Of the 30 subjects, one female dropped out of the study because of an adverse event (sudden deafness deemed by the medical investigator as unrelated to supplementation); this subject's data were excluded from the efficacy assessment. The remaining 29 subjects were deemed eligible for efficacy assessment and their baseline characteristics are shown in Table 1.

Table 2 shows the changes in functions of locomotor system including the knee joint over the 16-week intervention period. The VAS score for JKOM knee pain, JKOM total score, GLFS-5 score, and all VAS scores for knee pain in various daily situations significantly decreased at week 4 and thereafter $(P<0.05$ or $P<0.01)$ compared with the baseline. The questionnaire scores reflecting frequency of tripping while walking or ascending/descending stairs significantly decreased at week 8 and thereafter $(P<0.05$ or $P<0.01)$ compared with the baseline.

Table 3 shows the changes in parameters analyzed by the motion capture system over the 16-week intervention period. Normal walking speed significantly increased at week 16 compared with the baseline $(P<0.01)$. Step length and stride length significantly increased at week 8 and at week 16 compared with the baseline $(P<0.05, P<0.01)$, but cadence was not significantly changed by the intervention. Time in the stance phase significantly decreased at week 8 and at week 16 compared with the baseline $(P<0.01)$, but time in the swing phase was not significantly changed by

Table I Baseline characteristics of subjects eligible for efficacy assessment

\begin{tabular}{ll}
\hline Variables & Values $^{\mathrm{a}}(\mathbf{n}=\mathbf{2 9})$ \\
\hline Sex (male:female) & $14: 15$ \\
Age (years) & $56.9 \pm 1.9$ \\
Height $(\mathrm{cm})$ & $161.7 \pm \mathrm{I} .7$ \\
Body weight $(\mathrm{kg})$ & $62.6 \pm 2.1$ \\
Body mass index $\left(\mathrm{kg} / \mathrm{m}^{2}\right)$ & $23.8 \pm 0.6$ \\
Systolic blood pressure $(\mathrm{mmHg})$ & $120.4 \pm 2.5$ \\
Diastolic blood pressure $(\mathrm{mmHg})$ & $73.3 \pm 1.6$ \\
Heart rate (beats/min) & $72.0 \pm 2.4$ \\
JOA criteria aggregate scores $\left(\right.$ points) ${ }^{\mathrm{b}}$ & $165.9 \pm 3.4$ \\
K-L grades $(0, \mathrm{I}, \mathrm{II})$ & $2,22,5$ \\
\hline
\end{tabular}

Notes: aAll values except sex and $\mathrm{K}-\mathrm{L}$ grades are expressed as mean \pm standard error. 'Values are expressed as summed data for both knees.

Abbreviations: JOA, Japanese Orthopaedic Association; K-L, Kellgren-Lawrence. intervention. Angle of soles at the end of the stance phase significantly increased at week 8 and at week 16 compared with the baseline $(P<0.05, P<0.01)$ and angle of toe at the beginning of the stance phase significantly increased at week 16 compared with the baseline $(P<0.01)$.

Table 4 shows correlations between changes in major parameters analyzed by the motion capture system and locomotor functions that involve the knee joint over the 16-week intervention period. Normal walking speed significantly correlated with stride length, cadence, and angle of sole $(P<0.01, r=0.87 ; P<0.05, r=0.44$; and $P<0.01$, $r=0.66$, respectively). Stride length correlated with angle of sole at the end of the stance phase and with angle of toe at the beginning of the stance phase $(P<0.01, r=0.66 ; P<0.05$, $r=0.42$, respectively). The VAS score for JKOM knee pain, JKOM total score, and GLFS-5 significantly correlated with each other. JKOM total score and GLFS-5 significantly correlated with stride length $(P<0.05, \rho=0.42 ; P<0.05$, $\rho=-0.42$, respectively).

\section{Discussion}

The present study was conducted to investigate the biomechanical mechanism for GCQID's positive effects on locomotor functions in subjects with knee pain using a motion capture system.

GCQID improved functions of locomotion that involve the knee joint, as evidenced by JKOM and GLFS-5 scores (Table 2), suggesting that GCQID supplementation exerted effects on knee pain in daily life and on QOL, in accordance with our previous study. ${ }^{11}$ The motion capture analysis revealed that GCQID simultaneously increased normal walking speed and stride (and step) length, but that cadence was not changed (Table 3). As it has been reported that walking speed is mainly controlled by stride length and cadence, ${ }^{16}$ GCQID's effect of increasing normal walking speed was likely attributable to increased stride length. This attribution is supported by the fact that the change in normal walking speed at 16 weeks was most strongly related to the change in stride length in the correlation analysis $(r=0.87)$. In addition, GCQID increased the angle of soles at the end of the stance phase (Table 3), which could indicate increased kicking force of the ground and enhanced driving power (Figure 1). As the change in stride length at 16 weeks was correlated with the change in the angle of soles at the end of the stance phase $(r=0.66)$, we considered that GCQID might increase stride length through enhanced driving power. OA patients with knee pain had shorter stride lengths compared with healthy subjects, ${ }^{6,7}$ and the driving 
Table 2 Changes in functions of locomotor system including the knee joint over the 16 -week intervention period

\begin{tabular}{|c|c|c|c|c|c|}
\hline \multirow[t]{2}{*}{ Variables } & \multicolumn{5}{|c|}{ Values at each time point $(n=29)$} \\
\hline & Baseline & Week 4 & Week 8 & Week I 2 & Week 16 \\
\hline \multicolumn{6}{|l|}{ JKOM } \\
\hline (I) VAS score for knee pain (mm) & $59.7 \pm 3.8$ & $30.9 \pm 3.9 * *$ & $25.2 \pm 3.8 * *$ & $25.9 \pm 4 . I^{* *}$ & $16.0 \pm 3.5^{* *}$ \\
\hline (II-V) Total score (points) & $38.0 \pm 2.5$ & $23.6 \pm 2.2 * *$ & $20.9 \pm 2.4 * *$ & $19.7 \pm 2.2 * *$ & $15.9 \pm 2.0 * *$ \\
\hline GLFS-5 (points) & $9.2 \pm 0.6$ & $6.5 \pm 0.6 *$ & $5.7 \pm 0.6 * *$ & $5.1 \pm 0.6 * *$ & $4.6 \pm 0.6 * *$ \\
\hline \multicolumn{6}{|l|}{ VAS score for knee pain in various daily situations } \\
\hline Pain (right knee) on rest (mm) & $26.9 \pm 5.4$ & $11.9 \pm 3.4^{* *}$ & $8.0 \pm 2.9 * *$ & $9.1 \pm 3.0 * *$ & $5.0 \pm 1.9 * *$ \\
\hline Pain (left knee) on rest (mm) & $23.4 \pm 4.7$ & $9.6 \pm 2.7 * *$ & $8.8 \pm 3.2^{* *}$ & $10.5 \pm 3.4 * *$ & $5.3 \pm 2.5 * *$ \\
\hline Pain (right knee) on walking (mm) & $45.4 \pm 5.3$ & $19.4 \pm 3.7 * *$ & $15.7 \pm 3.6 * *$ & $17.0 \pm 3.5 * *$ & $10.4 \pm 2.9 * *$ \\
\hline Pain (left knee) on walking (mm) & $39.0 \pm 5.3$ & $21.1 \pm 4.3 * *$ & $15.4 \pm 3.9 * *$ & $17.4 \pm 4.3 * *$ & $11.8 \pm 3.8 * *$ \\
\hline Pain (right knee) on ascending/descending stairs (mm) & $53.4 \pm 6.0$ & $31.7 \pm 5.3 * *$ & $24.6 \pm 4.7 * *$ & $23.7 \pm 4.7^{* *}$ & $13.3 \pm 3.1 * *$ \\
\hline Pain (left knee) on ascending/descending stairs (mm) & $45.6 \pm 5.4$ & $27.1 \pm 5.0 * *$ & $21.6 \pm 4.4^{* *}$ & $21.4 \pm 5.1 * *$ & $14.8 \pm 3.8 * *$ \\
\hline \multicolumn{6}{|l|}{ Questionnaire } \\
\hline Score for tripping while walking (points) & $2.7 \pm 0.2$ & $2.3 \pm 0.2$ & $2.1 \pm 0.2 *$ & $1.9 \pm 0.2 * *$ & $2.0 \pm 0.1 *$ \\
\hline Score for tripping while ascending/descending stairs (points) & $2.6 \pm 0.2$ & $2.1 \pm 0.2$ & $1.8 \pm 0.1 *$ & $1.8 \pm 0.2^{*}$ & $1.7 \pm 0.1 * *$ \\
\hline
\end{tabular}

Notes: Values are expressed as mean \pm standard error. $* P<0.05$; $* * P<0.01$ for comparisons with baseline.

Abbreviations: GLFS-5, 5-question Geriatric Locomotive Function Scale; JKOM, Japanese Knee Osteoarthritis Measure; VAS, visual analog scale.

power subjects needed to make longer strides could have been restricted because of knee pain. Because the change in stride length at 16 weeks was correlated with changes in JKOM total score and GLFS- 5 score $(\rho=-0.44$ and $\rho=-0.42$, respectively), GCQID might increase stride length through alleviated knee pain and improved functions of locomotor system in daily life. In addition, GCQID increased angle of toe at the beginning of the stance phase (Table 3), which could indicate that the manner of gait control of subjects was changed to one in which toes were raised higher (Figure 1). The change in angle of toe at the beginning of the stance phase at 16 weeks was correlated with the change in stride length $(r=0.42$ ), suggesting the possibility of a contribution of increased stride length to higher toe angle by GCQID. As GCQID decreased frequency of tripping while walking, according to the questionnaires (Table 2), GCQID might actually change the gait in a way that makes subjects less likely to be tripped.
There are some limitations to the present study. First, it was conducted as an open label study. Therefore, we cannot deny the possibility of a placebo effect as an explanation for the observed effect of GCQID. Although improvement of scores for functions of locomotor system, such as the JKOM total score, was comparatively greater than that in our previous study, the amount of improvement in normal walking speed by GCQID was very similar to that found for the GCQID group in our previous study. ${ }^{11}$ Therefore, we assert that GCQID likely produced a similar effect as in our previous study, in terms of walking parameters. This point will be more apparent as future randomized, placebo-controlled trials elucidating the effect of GCQID supplement on walking parameters are conducted. Second, although the biochemical mechanism of GCQID supplementation to improve locomotor functions may be explained mainly by alleviated knee pain through the anti-inflammatory and chondroprotective activities of glucosamine hydrochloride, ${ }^{17,18}$ chondroitin

Table 3 Changes in parameters analyzed by motion capture system over the 16-week intervention period

\begin{tabular}{|c|c|c|c|}
\hline \multirow[t]{2}{*}{ Variables } & \multicolumn{3}{|c|}{ Values at each time point $(n=29)$} \\
\hline & Baseline & Week 8 & Week 16 \\
\hline Normal walking speed (m/sec) & $0.98 \pm 0.05$ & $1.02 \pm 0.04$ & $1.10 \pm 0.04 * *$ \\
\hline Step length $(\mathrm{cm})$ & $52.81 \pm 2.22$ & $55.05 \pm 2.12 *$ & $58.18 \pm 1.85 * *$ \\
\hline Stride length $(\mathrm{cm})$ & $104.97 \pm 4.33$ & $109.54 \pm 4.17^{*}$ & $115.33 \pm 3.73^{* *}$ \\
\hline Cadence (steps/min) & $|34.94 \pm 2.8|$ & $135.59 \pm 2.59$ & $|35.32 \pm 3.0|$ \\
\hline Time during stance phase (sec) & $0.67 \pm 0.02$ & $0.64 \pm 0.01 * *$ & $0.63 \pm 0.01 * *$ \\
\hline Time during swing phase (sec) & $0.45 \pm 0.01$ & $0.45 \pm 0.01$ & $0.45 \pm 0.01$ \\
\hline Angle of sole at the end of the stance phase (deg) & $36.01 \pm 1.06$ & $38.25 \pm 0.93 *$ & $39.47 \pm 0.84 * *$ \\
\hline Angle of toe at the beginning of the stance phase (deg) & $17.52 \pm 0.82$ & $17.46 \pm 0.92$ & $19.02 \pm 0.88 * *$ \\
\hline
\end{tabular}

Notes: Values are expressed as mean \pm standard error. $* P<0.05$; $* * P<0.01$ compared with baseline value. 
Table 4 Correlations between changes in major parameters analyzed by motion capture system and locomotor functions that involve the knee joint over the I6-week intervention period

\begin{tabular}{|c|c|c|c|c|c|c|c|c|}
\hline & $\begin{array}{l}\text { Normal } \\
\text { walking speed }\end{array}$ & Stride length & Cadence & Angle of sole & Angle of toe & $\begin{array}{l}\text { VAS score } \\
\text { for JKOM }\end{array}$ & $\begin{array}{l}\text { JKOM } \\
\text { total score }\end{array}$ & GLFS-5 \\
\hline \multicolumn{9}{|l|}{ Normal walking speed } \\
\hline Stride length & $0.866 * *$ & & & & & & & \\
\hline Cadence & $0.440^{*}$ & 0.229 & & & & & & \\
\hline Angle of sole & $0.656 * *$ & $0.665^{* *}$ & 0.232 & & & & & \\
\hline Angle of toe & 0.326 & $0.418^{*}$ & -0.083 & -0.111 & & & & \\
\hline VAS score for JKOM & -0.117 & -0.131 & 0.024 & -0.194 & 0.103 & & & \\
\hline JKOM total score & -0.263 & $-0.440 *$ & 0.026 & -0.234 & 0.007 & $0.49 I^{* *}$ & & \\
\hline GLFS-5 & -0.300 & $-0.420 *$ & 0.044 & -0.133 & 0.052 & $0.599 * *$ & $0.768 * *$ & \\
\hline
\end{tabular}

Notes: Values are expressed as Pearson's correlation coefficient ( $r$ value) for parametric variables and Spearman's rank correlation coefficient ( $\rho$ value) for nonparametric variables. $* P<0.05 ; * * P<0.01$.

Abbreviations: GLFS-5, 5-question Geriatric Locomotive Function Scale; JKOM, Japanese Knee Osteoarthritis Measure; VAS, visual analog scale.

sulfate, ${ }^{19}$ and quercetin, ${ }^{20,21}$ in addition to direct effects on muscle such as anti-muscle atrophy effects of quercetin, ${ }^{22}$ and the effect of imidazole peptides on increasing muscle blood flow, ${ }^{23}$ as described in our previous study, ${ }^{11}$ the contribution of each component to the effects on locomotor functions in this study and our previous study have not been determined. Further studies are needed in the future to elucidate the precise role of each component. Third, the present study was a short-term study of 16 weeks with a small number of subjects, not enough to elucidate the influence of characteristics of subjects such as age and sex. Therefore, long-term studies with larger sample size are required in the future for assessing the efficacy and safety of GCQID treatment including age- and sex-specific variations more clearly.

\section{Conclusion}

Our data based on gait analysis using a motion capture system suggest that GCQID supplements can increase walking speed through increased stride length and increased force of kicking from the ground during steps, and these improvements may be associated mainly with alleviated knee pain and direct effects on muscle.

\section{Acknowledgments}

We thank all participants and professional staff at the School of Physical Education at Tokai University for their support of the study.

\section{Disclosure}

NK, YO, TI, and HS are employed by Suntory Wellness Ltd, which is the sponsor of this study and markets health food products, such as the GCQID used in this study. The authors report no other conflicts of interest in this work. This work was presented at the World Congress on Osteoporosis, Osteoarthritis and Musculoskeletal Diseases (WCO-IOF-ESCEO 2015) held March 26-29, 2015 in Milan, Italy and the abstract was published in Osteoporos Int. 2015;26(Suppl 1):S97.

\section{References}

1. Nakamura K. A "super-aged" society and the "locomotive syndrome". J Orthop Sci. 2008;13(1):1-2.

2. Japanese Ministry of Health, Labour and Welfare. The second term of National Health Promotion Movement in the 21st Century (Health Japan 21 (the second term)) 2012. Available from: http://www.mhlw. go.jp/file/06-Seisakujouhou-10900000-Kenkoukyoku/0000047330.pdf. Accessed August 27th, 2015.

3. Seichi A, Hoshino Y, Doi T, Akai M, Tobimatsu Y, Iwaya T. Development of a screening tool for risk of locomotive syndrome in the elderly: the 25-question Geriatric Locomotive Function Scale. J Orthop Sci. 2012;17(2):163-172.

4. Yoshimura N, Muraki S, Oka H, et al. Prevalence of knee osteoarthritis, lumbar spondylosis, and osteoporosis in Japanese men and women: the research on osteoarthritis/osteoporosis against disability study. J Bone Miner Metab. 2009;27(5):620-628.

5. Yoshimura N, Akune T, Fujiwara S, et al. Prevalence of knee pain, lumbar pain and its coexistence in Japanese men and women: The Longitudinal Cohorts of Motor System Organ (LOCOMO) study. J Bone Miner Metab. 2014;32(5):524-532.

6. Levinger P, Lai DT, Menz HB, et al. Swing limb mechanics and minimum toe clearance in people with knee osteoarthritis. Gait Posture. 2012;35(2):277-281.

7. Taş S, Güneri S, Baki A, Yıldırım T, Kaymak B, Erden Z. Effects of severity of osteoarthritis on the temporospatial gait parameters in patients with knee osteoarthritis. Acta Orthop Traumatol Turc. 2014; 48(6):635-641.

8. McAlindon TE, LaValley MP, Gulin JP, Felson DT. Glucosamine and chondroitin for treatment of osteoarthritis: a systematic quality assessment and meta-analysis. JAMA. 2000;283(11):1469-1475.

9. Reginster JY, Deroisy R, Rovati LC, et al. Long-term effects of glucosamine sulphate on osteoarthritis progression: a randomized, placebo-controlled clinical trial. Lancet. 2001;357(9252): 251-256.

10. Kanzaki N, Saito K, Maeda A, et al. Effect of a dietary supplement containing glucosamine hydrochloride, chondroitin sulfate and quercetin glycosides on symptomatic knee osteoarthritis: a randomized, double-blind, placebo-controlled study. J Sci Food Agric. 2012;92(4): 862-869. 
11. Kanzaki N, Ono Y, Shibata H, Moritani T. Glucosamine-containing supplement improves locomotor functions in subjects with knee pain: a randomized, double-blind, placebo-controlled study. Clin Interv Aging. 2015;10:1743-1753.

12. Tanaka S, Murata S, Iwanaga K, et al. Study of combined effect by thermotherapy and kinesitherapy on osteoarthritis of the knee. Japanese Journal of Health Promotion and Physical Therapy. 2011;1(2):131-135. Available from: https://www.jstage.jst.go.jp/article/hppt/1/2/1_131/_pdf. Accessed August 27th, 2015.

13. The Japanese Orthopaedic Association Japanese Knee Society. Assessment Criteria for Knee Diseases and Treatments. Tokyo: Kanehara; 1994.

14. Akai M, Doi T, Fujino K, Iwaya T, Kurosawa H, Nasu T. An outcome measure for Japanese people with knee osteoarthritis. J Rheumatol. 2005;32(8):1524-1532.

15. Kellgren JH, Lawrence JS. Radiological assessment of osteoarthritis. Ann Rheum Dis. 1957;16(4):494-502.

16. Egerton T, Danoudis M, Huxham F, Iansek R. Central gait control mechanisms and the stride length - cadence relationship. Gait Posture. 2011;34(2):178-182.

17. Fenton JI, Chlebek-Brown KA, Peters TL, Caron JP, Orth MW. Glucosamine $\mathrm{HCl}$ reduces equine articular cartilage degradation in explant culture. Osteoarthritis Cartilage. 2000;8(4):258-265.
18. Gouze JN, Bordji K, Gulberti S, et al. Interleukin-1beta down-regulates the expression of glucuronosyltransferase I, a key enzyme priming glycosaminoglycan biosynthesis: influence of glucosamine on interleukin-1beta-mediated effects in rat chondrocytes. Arthritis Rheum. 2001; 44(2):351-360.

19. Iovu M, Dumais G, du Souich P. Anti-inflammatory activity of chondroitin sulfate. Osteoarthritis Cartilage. 2008;16 Suppl 3:S14-S18.

20. Jackson JK, Higo T, Hunter WL, Burt HM. The antioxidants curcumin and quercetin inhibit inflammatory processes associated with arthritis. Inflamm Res. 2006;55(4):168-175.

21. Mamani-Matsuda M, Kauss T, Al-Kharrat A, et al. Therapeutic and preventive properties of quercetin in experimental arthritis correlate with decreased macrophage inflammatory mediators. Biochem Pharmacol. 2006;72(10):1304-1310.

22. Mukai R, Nakao R, Yamamoto H, Nikawa T, Takeda E, Terao J. Quercetin prevents unloading-derived disused muscle atrophy by attenuating the induction of ubiquitin ligases in tail-suspension mice. J Nat Prod. 2010;73(10):1708-1710.

23. Horii Y, Fujisaki Y, Fuyuki R, Nagai K. L-Carnosine's dose-dependent effects on muscle sympathetic nerves and blood flow. Neurosci Lett. 2015;591:144-148.
Clinical Interventions in Aging

\section{Publish your work in this journal}

Clinical Interventions in Aging is an international, peer-reviewed journal focusing on evidence-based reports on the value or lack thereof of treatments intended to prevent or delay the onset of maladaptive correlates of aging in human beings. This journal is indexed on PubMed Central, MedLine,

\section{Dovepress}

CAS, Scopus and the Elsevier Bibliographic databases. The manuscript management system is completely online and includes a very quick and fair peer-review system, which is all easy to use. Visit http://www.dovepress. com/testimonials.php to read real quotes from published authors. 